\title{
A Theoretical Review of Axion ${ }^{a}$
}

\author{
Jihn E. Kim \\ Department of Physics and Center for Theoretical Physics \\ Seoul National University, Seoul 151-742, Korea
}

\begin{abstract}
It is emphasized that the existence of a very light axion is consistent with the strong CP invariance and cosmological and astrophysical constraints. The attempt to embed the very light axion in superstring models is discussed.
\end{abstract}

\section{The Strong CP Problem}

The standard model $S U(3) \times S U(2) \times U(1)$ describes the weak and electromagnetic interactions very successfully. The strong interaction part, quantum chromodynamics, is proven to be successful at perturbative level, but the study of nonperturbative effects are not so successful. Because of the lack of a calculational tool of the nonperturbative effects, the frequently used method for the study of QCD at low energy is the symmetry principle. At energy scales below the confinement and chiral symmetry breaking scale, some symmetries of QCD are manifest in strong interaction dynamics. Baryon number is known to be conserved. Chiral symmetry is broken.

Discrete symmetries are believed to be conserved in the process of confinement. Here we are interested in CP. If QCD conserves CP, the CP symmetry will be preserved in strong interactions at low energy. If $\mathrm{QCD}$ violates $\mathrm{CP}$, its effect will be shown in low energy strong interaction dynamics. QCD before 1975 was described by

$$
\mathcal{L}=-\frac{1}{2 g^{2}} \operatorname{Tr} F_{\mu \nu} F^{\mu \nu}+\bar{q}\left(i \not D-M_{q}\right) q
$$

where $q$ and $M_{q}$ are quark and quark mass matricies, respectively. After the discovery of the instanton solution in non-Abelian gauge theories it is known that the $\theta$-term must be considered

$$
\mathcal{L}_{\theta}=\frac{\theta}{16 \pi^{2}} \operatorname{Tr} F_{\mu \nu} \tilde{F}^{\mu \nu} .
$$

Note that $\mathcal{L}_{\theta}$ is odd uner $\mathrm{P}$ or $\mathrm{T}$ discrete transformation. Therefore, this term violates the $\mathrm{CP}$ invariance. Thus QCD contains a term violating $\mathrm{CP}$ symmetry. If $\mathrm{QCD}$ violates the $\mathrm{CP}$ symmetry, it must be revealed in strong interaction dynamics. For example, we may expect a $\mathrm{CP}$ violating static property of

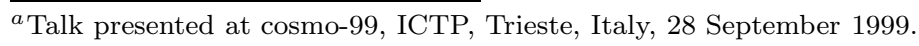


neutron, the electric dipole moment of neutron $d_{n}$. The experimental upper limit of $d_{n}$ is known to be $0.63 \times 10^{-25} e \cdot \mathrm{cm}^{3}$ On the other hand, if strong interaction violates the $\mathrm{CP}$ invariance at the full strength, then we expect $d_{n} \sim 10^{-14} e \cdot \mathrm{cm}$. Therefore, the vacuum angle is restricted to a tiny region

$$
|\theta|<10^{-9} .
$$

The question, "Why is the vacuum angle so small", is the strong CP problem. If we treat $\theta$ as $\mathrm{O}(1)$ parameter, then the above observation excludes QCD as the theory of strong interactions. In this case, different $\theta$ 's describe different universes. Since QCD is known to be so successful except for the strong CP problem, it is better to keep QCD as the theory of strong interactions. It is desirable to resolve the strong $\mathrm{CP}$ problem with QCD untouched.

There have been several attempts toward the solution of the strong CP problem, one even incorrectly asserting that there is no strong CP problem A $^{-}$

One class of solutions employs CP symmetry at the Lagrangian level, and require the induced vacuum angle (in the process of introducing weak CP violation) sufficiently smalls These are called natural solutions. Here, the weak CP violation is through spontaneous symmetry breaking or soft breaking. Namely, the Kobayashi-Maskawa type weak CP violation is not possible except for the Nelson-Barr type. The reason for a possible Kobayashi-Maskawa weak CP violation in the Nelson-Barr type strong CP solution is that the spontaneous $\mathrm{CP}$ violation here is introduced at a super high energy scale and hence at the electroweak scale CP is already violated as in the Kobayashi-Maskawa model. In this class of models, at tree level $\theta=0$, which implies $\operatorname{Arg} \operatorname{Det} M_{q}=0$. This is attained by assuming a CP invariant Lagrangian including the $\theta$ term and specific symmetries. Possible symmetries used for this purpose are: left-right symmetry, $U(1)$ gauge symmetry, permutation symmetry, and other discrete symmetries.

The other solutions are the $m_{u}=0$ solution and the axion solution.

Here, the most attractive solution is the axion solution, making $\theta$ a dynamical variable. A dynamical $\theta$ is equivalent to a pseudoscalar field which we call axion. If the axion has a potential, then in the evolving universe the minimum of the vacuum will be chosen. The axion solution guarantees that $\theta=0$ is the minimum of the $\theta$ potential, which is discussed below.

${ }^{b}$ This reference [4] starts with an assumption on CPT invariant $|n\rangle$ vacua and obtains the CPT odd $|\theta\rangle$ vacuum. But in Yang-Mills theories, $|n\rangle$ vacua are not CPT invariant, and the $|\theta\rangle$ vacuum is CPT invariant. 


\section{The Peccei-Quinn Solution}

The axion solution is a dynamical solution. Peccei and Quinn 6 showed that $\theta=0$ at the point $d V / d \theta=0$, which has been shown later by Vafa and Wittent

$$
\mathcal{L}=-\frac{1}{4} F^{2}+\bar{q}\left(i \not D-M_{q}\right) q+\theta \frac{g^{2}}{32 \pi^{2}} F \tilde{F}
$$

where we suppressed the Greek indices for space-time in $F$. Let us treat $\theta$ as a coupling. After integrating the quark fields out, the generating functional in the Euclidian space is given by

$$
\int\left[d A_{\mu}\right] \prod_{i} \operatorname{Det}\left(\not D+m_{i}\right) \exp \left\{-\int d^{4} x\left[\frac{1}{4 g^{2}} F^{2}-i \theta \frac{1}{32 \pi^{2}} F \tilde{F}\right]\right\} .
$$

Note that the resulting functional has a specific form of the $\theta$ dependence. In the Euclidian space, corresponding to the eigenstate $\psi$ of $i \not D, i \not D \psi=\lambda \psi$, there corresponds to the other eigenstate of $i \not D, i \not D\left(\gamma_{5} \psi\right)=-\lambda\left(\gamma_{5} \psi\right)$. Thus, the nonzero real eigenvalues of $i \not D$ are paired with opposite sign and the identical magnitude. For $N_{0}$ number of zero modes, we can show that

$$
\operatorname{Det}\left(\not D+m_{i}\right)=\prod_{i}\left(-i \lambda+m_{i}\right)=m_{i}^{N_{0}}\left(m_{i}^{2}+\lambda^{2}\right)>0 .
$$

Thus, the generating functional is bounded by usung the Schwarz inequality in view of Eq. (6),

$$
\begin{gathered}
\exp \left[-\int d^{4} x V[\theta]\right] \equiv\left|\int\left[d A_{\mu}\right] \prod \operatorname{Det}\left(\not D+m_{i}\right) \exp \left(-\int d^{4} x \mathcal{L}\right)\right| \\
\leq \int\left[d A_{\mu}\right]\left|\prod \operatorname{Det}\left(\not D+m_{i}\right) \exp \left(-\int d^{4} x \mathcal{L}\right)\right| \\
=\left|\int\left[d A_{\mu}\right] \prod \operatorname{Det}\left(\not D+m_{i}\right) \exp \left[-\int d^{4} x \mathcal{L}(\theta=0)\right]\right| \\
=\exp \left(-\int d^{4} x V[0]\right)
\end{gathered}
$$

where $\mathcal{L}=\left(1 / 4 g^{2}\right) F^{2}-i \theta\{F \tilde{F}\}$, and the simplified notation \{\} includes a factor $1 / 32 \pi^{2}$. The above inequality guarantees

$$
V[\theta] \geq V[0] .
$$

Instanton solutions have integer values for $\int d^{4} x\{F \tilde{F}\}$, hence $V[\theta]$ is periodic with the $\theta$ period of $2 \pi, \theta \rightarrow \theta+2 n \pi$. The above agument is for a nonzero up quark mass. If $m_{u}=0, \theta$ is unphysical and there is no strong CP problem.

As a coupling, any $\theta$ defines a good theory (or universe). The shape of $V$ as a function of $\theta$ is 


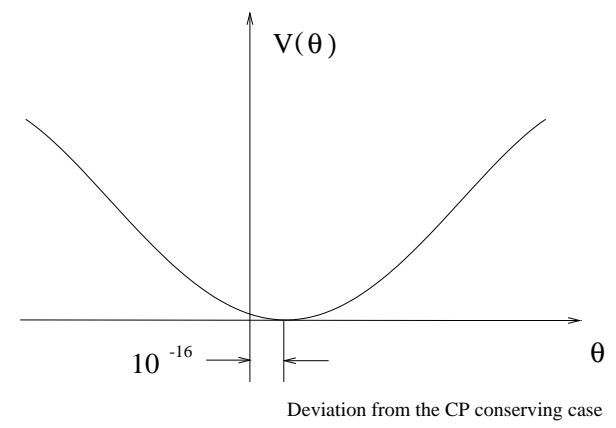

Fig. 1. Shape of $V[\theta]$. The KM weak $C P$ introduces $\mathrm{B}|\theta| \simeq 10^{-16}$.

Here, the axion solution of the strong $\mathrm{CP}$ problem is transparent. If we identify $\theta$ as a pseudoscalar field,

$$
\theta=\frac{a}{F_{a}}
$$

the vacuum angle is chosen at $\theta \simeq 0$, realizing the almost $\mathrm{CP}$ invariant $\mathrm{QCD}$ vacuum. Note the key ingredients of this axion solution. Firstly, the theory introduces an axion coupling $a\{F \tilde{F}\}$. Second, there is no axion potential except that coming from the $F \tilde{F}$ term, otherwise our proof does not go through. Third, there necessarily appears a mass parameter $F_{a}$, the so-called axion decay constant. One possible example for the axion is to introduce a Goldstone boson, using a global $U(1)$ symmetry. 6 As explained above Peccei-Quinn showed that $\theta=0$ is the minimum of the potential, which is meaningful only if there is a dynamical field $a$. Later Weinberg and Wilczek explicitly showed that the model contains the axion. 9 It was immediately known that the PQWW axion does not exist 10 and hence there soon appeared a flurry of natural solutions.

To have the anomalous coupling, the global symmetry must have a triangle anomaly in $U(1)_{\text {global }} \times S U(3)_{c} \times S U(3)_{c}$. This anomalous coupling introduces an axion decay constant which can be large for the very light axion models 11.12 Soon after the invention of the very light axion, it has been known that the astrophysical and cosmological bounds restrict the axion decay constant in the region, $10^{9} \mathrm{GeV} \leq F_{a} \leq 10^{12} \mathrm{GeV}^{13}$. 14 Also, it was known that a more ambitious composite axion can be constructed 15

Another interesting possibility is that there results an axion with the above properties from a more fundamental theory such as from the string theory. This possibility introduces a nonrenormalizable interaction $a F \tilde{F}$. Indeed, superstring models have a host of moduli fields which do not have potentials at 
the compactification scale. But some of the moduli have the desired anomalous couplings 16 and becomes the axion. In this case, the so-called superstring axions are expected to have the decay constant near the Planck scale. But the exact magnitude depends on the details of the model.

The mass of the very light axion is an important parameter in the evolving universe. The allowed range of the decay constant gives tens of micro-eV axion mass,

$$
m_{a}=0.6 \times 10^{7} \frac{\mathrm{eV}}{F_{a}^{\mathrm{GeV}}}
$$

where $F_{a}^{\mathrm{GeV}}=F_{a} / \mathrm{GeV}$.

The neutron electric dipole moment in the $\theta$ vacuum is given by 17

$$
\frac{d_{n}}{e}=O(1) \frac{m_{u} \sin \theta}{f_{\pi}^{2}\left[2 Z \cos \theta+(1+Z)^{2}\right]^{1 / 2}}
$$

where $Z=m_{u} / m_{d}$ is the ratio of the current quark masses. For $m_{u}<2 \times$ $10^{-13} \mathrm{GeV}$, the neutron electric dipole moment is satisfied even for $\mathrm{O}(1) \theta$.

The very light axion physics is closely connected to the study of the evolution of the universe. The domain wall problem 18 must be studied in specific models. Usually, inflation needed in supergravity models with the condition on the reheating temperature $T_{R}<10^{9} \mathrm{GeV} 19$ does not lead to the axionic domain wall problem.

Theoretically, the introduction of the Peccei-Quinn U(1) global symmetry is ad hoc. It is better if the axion arises from a fundamental theory. In this spirit, it is most important to draw a very light axion from superstring theory. If we cannot, how can we understand the strong $\mathrm{CP}$ problem?

\section{Embedding the Very Light Axion in Superstring}

The pseudoscalar moduli fields in $\mathrm{D}=10$ superstring is $B_{M N}(M, N=0, \cdots, 9)$ among the bosonic fields $G_{M N}, B_{M N}$ and the dilaton. Upon compactification to $\mathrm{D}=4, B_{\mu \nu}(\mu, \nu=0, \cdots, 3)$ turns out to be a pseudoscalar field. Dual transformation of $B_{\mu \nu}$ defines a pseudoscalar $a$ as

$$
\partial^{\sigma} a \propto \epsilon^{\mu \nu \rho \sigma} H_{\mu \nu \rho} ; H_{\mu \nu \rho}=\text { field strength of } B_{\mu \nu} \propto \epsilon_{\mu \nu \rho \sigma} \partial^{\sigma} a .
$$

Of course, $B_{\mu \nu}$ does not have renormalzable couplings to matter fields, hence there is no potential for $a$ since the possible derivative coupling $\partial^{\mu} a \psi \gamma_{\mu} \gamma_{5} \psi$ does not lead to a potential term. If we consider a field strenth $H_{\mu \nu \rho}$ to obtain couplings, it is invariant under a shift $a \rightarrow a+c$. This consideration does not lead to an anomalous coupling needed for an axion. 
Hoyever, the gauge invariant $\mathrm{D}=10$ field strength of $B_{M N}$ is not $H=d B$, 9 but is 20

$$
H=d B+\omega_{3 Y}^{0}-\omega_{3 L}^{0}
$$

where the Yang-Mills Chern-Simmons form is $\operatorname{tr}\left(A F-A^{3} / 3\right)$ and the Lorentz Chern-Simmons form is $\operatorname{tr}\left(\omega R-\omega^{3} / 3\right)$. The Chern-Simmons forms satisfy $d \omega_{3 Y}^{0}=\operatorname{tr} F^{2}$ and $d \omega_{3 L}^{0}=\operatorname{tr} R^{2}$. Therefore,

$$
d H=-\operatorname{tr} F^{2}+\operatorname{tr} R^{2},
$$

and the equation of motion for $a$ is

$$
\square a=-\frac{1}{M}\left[\operatorname{Tr} F_{\mu \nu} \tilde{F}^{\mu \nu}-\operatorname{Tr} R_{\mu \nu} \tilde{R}^{\mu \nu}\right]
$$

which implies $a F \tilde{F}$ coupling which is needed for the axion interpretation of $a 10$ This is the so-called model-independent axion. Here, $M$ is about the compactification scale suppressed by a factor and corresponds to the axion decay constant 21

Ta cancel the Yang-Mills anomaly, one should introduce the Green-Schwarz term $20 S_{G S} \sim \int\left(B \operatorname{tr} F^{4}+\cdots\right)$. The Green-Schwarz term gives the needed anomalous coupling for pseudoscalars $B_{i j}(i, j=4, \cdots, 9)$

$$
B_{i j} \epsilon^{\mu \nu \rho \sigma} F_{\mu \nu} F_{\rho \sigma}\left\langle F_{k l}\right\rangle\left\langle F_{p q}\right\rangle \epsilon^{i j k l p q} .
$$

Here, we have model-dependent axions $a_{k} \sim \epsilon_{i j k} B_{i j}$, the number of which is the second Betti number 22 Unlike the model-independent axion, the modeldependent axions receive nonvanishing superpotential terms from the worldsheet instanton effect,

$$
\int_{\Sigma_{J}} d^{2} z \omega_{i j}^{I}\left(\partial X^{i} \underline{\partial} X^{\underline{j}}-\underline{\partial} X^{i} \partial X^{\underline{j}}\right)=2 \alpha^{\prime} \delta_{I J}
$$

where $\alpha^{\prime}$ is the string tension, and $\omega=4 \pi^{2} \operatorname{Re}\left(T_{I}\right) \omega^{I}$. The internal space volume is given by ${ }^{23} V_{6}=(1 / 3 !) \int \omega \wedge \omega \wedge \omega \approx(1 / 6)\left(4 \pi^{2} \operatorname{Re} T\right)^{3}\left(2 \alpha^{\prime}\right)^{3}$. So the model-dependent axion cannot be a candidate for the low energy QCD axion unless the potential is sufficiently suppressed 24

Thus, the model-independent axion is a good candidate for the QCD axion. However, it has two serious problems:

(A) The decay constant problem- $F_{a}$ is too large, $\sim 10^{16} \mathrm{GeV} 11$ and

(B) The hidden sector problem- We need a hidden sector confining force for supersymmetry breaking around $10^{10-13} \mathrm{GeV}$. If so, the dominant contribution to the model-independent axion comes from the hidden sector anomaly,

${ }^{c}$ In this paragraph we use the differential form. 


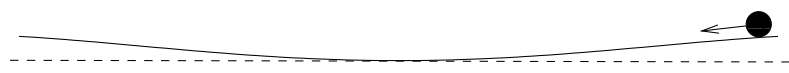

Fig. 2. The almost flat axion potential.

$m_{a} \simeq \Lambda_{h}^{2} / F_{a}$. Then, this cannot be the needed axion for the strong CP problem. With two confining forces with scales of $\Lambda_{h}$ and $\Lambda_{Q C D}$, the potential can be written as

$$
V \sim-\Lambda_{Q C D}^{4} \cos (\theta+\alpha)-\Lambda_{h}^{4} \cos \left(\theta_{h}+\beta\right)
$$

where $\alpha$ and $\beta$ are constants, and $\Lambda_{h} \gg \Lambda_{Q C D}$. To settle both $\theta_{h}$ and $\theta$ dynamically at zero, we need two axions. But as shown above, we have only one axion for this purpose, the model-independent axion.

Both of the above problems are difficult to circumvent. [f

The approximate global symmetries may be a way out from this dilemma. 26 Discrete symmetries may forbid sufficiently many terms so that the PecceiQuinn symmetry violating terms can appear only at $d \geq 9$. One such example is $Z_{N}$ symmetry (e.g. $N=3$ ) in theories without hidden sector quarks 27

\section{Cosmology with Axion}

In the hot cores of stellar objects, the axion production can occur through $\gamma+e($ or $Z) \rightarrow a+e($ or $Z), n+n \rightarrow n+n+a, \gamma+e \rightarrow a+e, e^{+}+e^{-} \rightarrow a+\gamma$, etc. For a sufficiently large $F_{a}$, axions produced in the stellar core can escape the star since the rescattering cross section is small. If its production rate is too large $\left(\sim 1 / F_{a}^{2}\right)$, it takes out too much energy from the core. Thus, there results the upper bounds on $F_{a}$ from star evolutions. The best bound is obtained from the study of supernovae. $22,13,29$ Thus, the solar axion search may not succeed which needs $F_{a} \sim 10^{7} \mathrm{GeV}$.

The lifetime of $a$ is extremely long and hence can be treated in most cases as a stable particle. The classical coherent states of $a$ will oscillate around the minimum $\langle a\rangle=0$. When can this happen? It is around $T_{1} \simeq 1 \mathrm{GeV}$, not around the axion scale of $F_{a}$ since the axion potential is extremely flat. Its existence is felt when the expansion rate is smaller than the oscillation rate of the classical axion field, viz. $3 H<m_{a} 14$ For $T<T_{1}$, the classical axion field $\langle a\rangle$ begins to roll down the hill. After this happens, the Hubble expansion is negligible and the $\langle a\rangle$ equation leads to a conserved $m_{a} A^{2}$ (where $A$ is the amplitude of the classical axion field) in the comoving volume. This coherent axion field carries energy density behaving like nonrelativistic particles and its

${ }^{d}$ See, however, Lalak et al 25 


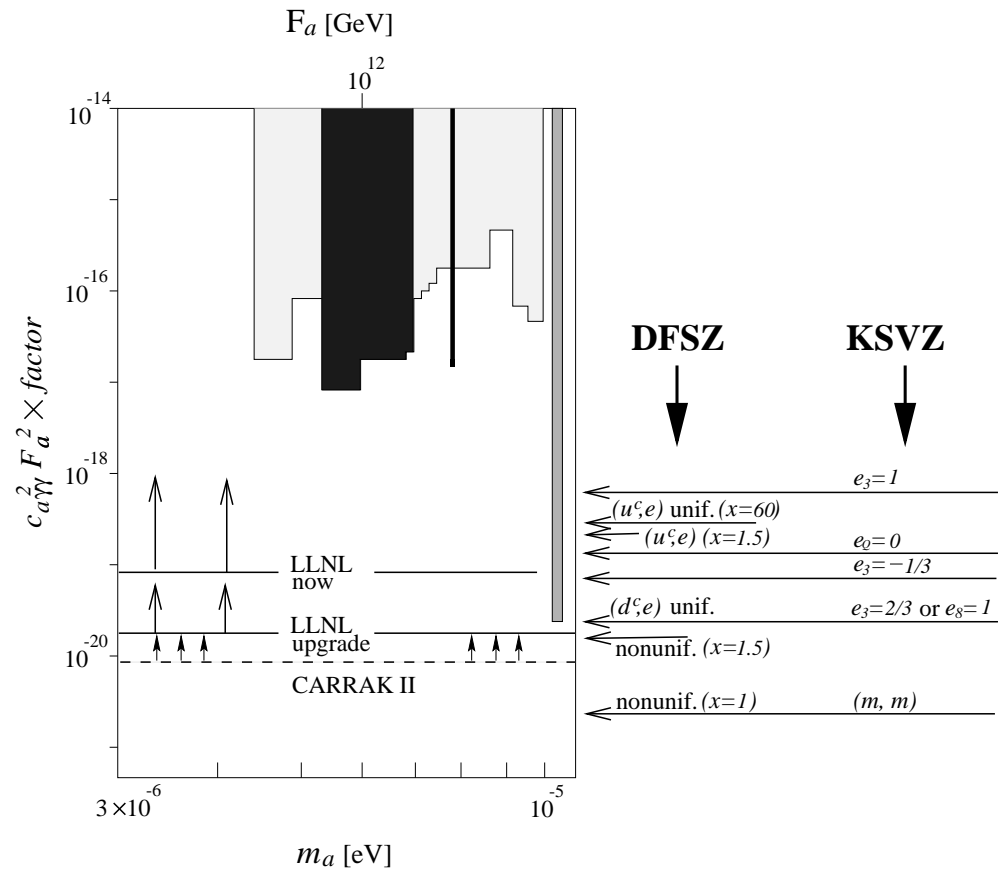

Fig. 3. Axion search experiments. The model predictions are shown 32

contribution to cosmic energy is 30

$$
\Omega_{a} h^{2} \simeq 0.13 \times 10^{ \pm 0.4} \Lambda_{200}^{-0.7} f\left(\theta_{1}\right)\left(\frac{10^{-5} \mathrm{eV}}{m_{a}}\right)^{1.18} N_{D W}^{2}
$$

where $\theta_{1}$ is the $\theta$ value at the cosmic temperature $T_{1}$. These considerations restrict $F_{a}$ as

$$
10^{9} \mathrm{GeV} \leq F_{a} \leq 10^{12} \mathrm{GeV} .
$$

In this scenario, cold axions are packed around us, for which the axion search experiments are perfprmed. In this search one probes the axion-electromagnetic coupling of $a \mathbf{E} \cdot \mathbf{B} 31$ The current status is exhibited in Fig. 3.

Depending on models, there can exist domain walls, but in supersymmetric models the requirement of $T_{R H}<10^{9} \mathrm{GeV}$ gives a sufficient dilution of the dangerous domain walls. Also there can exist hot axions produced by vibrations of axionic strings when they are formed around $T \sim F_{a} 33$ These hot axions are also diluted by inflation with $T_{R H}<10^{9} \mathrm{GeV}$. 


\section{Quintessence idea}

Recently, there is an evidence that the cosmological constant is very tiny 34 which is another difficult problem for the cosmological constant. Since the axion potential is almost flat, there may be a mechanism to have a very small cosmological constant within the axion idea. We start with the assumption that at the minimum of the potential the cosmological constant is zero.

We note that the massless quark solution of the strong CP problem leads to a flat $\theta_{h}$ direction. Eventually, we will identify this $\theta_{h}$ direction as the hidden sector(h-sector) axion direction. If we break the global symmetry by a tiny h-sector quark mass, the degeneracy is broken feebly. A random value of $\theta_{h}$ will give a generic value of the potential determined by the nonvanishing h-quark mass. This generic value of the potential energy is expected to be $(0.003 \mathrm{eV})^{4}$ so that it explains the Type 1a data 34 If $\theta_{h}$ is a coupling, then the random value of $\theta_{h}$ gives a true cosmological constant, i.e. it is zero. If $\theta_{h}$ is a dynamical field such as an axion, then the cosmological constant is nonzero like in axion models. We will call this dynamical $\theta_{h}$ with a currently interesting cosmological constant a quintessence. The axion quintessence needs a potential height of order $10^{-47} \mathrm{GeV}^{4}$ and $F_{a} \sim M_{P}$, i.e. $m_{a} \sim 10^{-33} \mathrm{eV}$, for it to dominate the mass density of the universe recently. In terms of the known scales, $M_{P}=2.44 \times 10^{18} \mathrm{GeV}$ and $v \simeq 247 \mathrm{GeV}$, we obtain a small energy density $v^{n+4} / M_{P}^{n}$. For $n=3$,

$$
\frac{v^{7}}{M_{P}^{3}} \sim 4 \times 10^{-39} \mathrm{GeV}^{4}
$$

which can be a reasonable candidate for the vacuum energy with a further suppression by coupling constants. How can one forbid $n=1,2$ but allow $n=3$ in supergravity?

With a (almost) massless h-quark, the h-sector instanton potential is most flat. Then the axion corresponding to the h-sector can be a quintessence 35 For this idea to work, we must introduce at least one model-dependent axion so that two axions survive. If two axions are present with $F_{1}$ and $F_{2}$ and two explicit scales $\Lambda_{1}$ and $\Lambda_{2}$ break the symmetries, the larger $F$ corresponds to the smaller $\Lambda$. Because the h-sector instanton potential is made almost flat by almost massless h-quark 35 the smaller symmetry breaking scale $\left(v^{7} / M_{P}^{3}\right)^{1 / 4}$ corresponds to the larger $F$, i.e. the Planck scale decay constant. This solves the axion decay constant problem by lowering the decay constant of the QCD axion to $10^{12} \mathrm{GeV}$.

Now the problem is how to save a model-dependent axion. For this we have to assume that many singlets do not develop vacuum expectation values 35 


\section{Conclusion}

We have shown that:

i) The strong CP problem is a serious problem.

ii) But there are solutions, natural 0 and automatic. 6.96,11, 12

iii) The very light axion solution is the most attractive one. 11.12 Here, the weak CP violation is of the Kobayashi-Maskawa type.

iv) The very light axion can close the universe 14 and can be detected.

v) Superstring models have two problems housing the very light axion. One possible scenario with quintessence is also discussed.

\section{Acknowledgments}

This work is supported in part by the Korea Research Foundation, Korea Science and Engineering Foundation, and the BK21 program of the Ministry of Education.

\section{References}

1. A. A. Belavin, A. M. Polyakov, A. S. Shvarts and Yu. S. Tyupkin, Phys. Lett. B59, 85 (1975).

2. C. G. Callan, R. Dashen and D. J. Gross, Phys. Lett. 63, 334 (1976); R. Jackiw and C. Rebbi, Phys. Rev. Lett. 37, 172 (1976).

3. P. G. Harris et al, Phys. Rev. Lett. 82, 904 (1999).

4. S. Okubo and R. E. Marshak, Prog. Theor. Phys. 87, 1059 (1992).

5. M. A. B. Beg and H.-S. Tsao, Phys. Rev. Lett. 41, 278 (1978); H. Georgi, Hadronic J. 1, 155 (1978); R. N. Mohapatra and G. Senjanovic, Phys. Lett. B79, 283 (1978); G. Segre and A. Weldon, Phys. Rev. Lett. 42, 1191 (1979); S. M. Barr and P. Langacker, Phys. Rev. Lett. 42, 1654 (1979); A. E. Nelson, Phys. Lett. B136, 387 (1984); S. M. Barr, Phys. Rev. Lett. 53, 329 (1984).

6. R. D. Peccei and H. R. Quinn, Phys. Rev. Lett. 38, 1448 (1977).

7. C. Vafa and E. Witten, Phys. Rev. Lett. 53, 535 (1983).

8. H. Georgi and L. Randall, Nucl. Phys. B276, 241 (1986).

9. S. Weinberg, Phys. Rev. Lett. 40, 223 (1978); F. Wilczek, Phys. Rev. Lett. 40, 279 (1978).

10. R. D. Peccei, in Proc. 19th ICHEP Meeting (Aug. 23-30, 1978), ed. H. Homma et al (Phys. Soc. of Japan, Tokyo, 1979), p.1045.

11. J. E. Kim, Phys. Rev. Lett. 43, 103 (1979); M. A. Shifman, A. I. Vainstein and V. I. Zakharov, Nucl. Phys. B166, 493 (1980). 
12. M. Dine, W. Fischler and M. Srednicki, Phys. Lett. B104, 199 (1981); A. P. Zhitnitskii, Sov. J. Nucl. Phys. 31, 260 (1980).

13. G. Raffelt and D. Seckel, Phys. Rev. Lett. 60, 1793 (1988); M. S. Turner, Phys. Rev. Lett. 60, 1797 (1988); Mayle et al, Phys. Lett. B203, 188 (1988).

14. J. Preskill, M. B. Wise and F. Wilczek, Phys. Lett. B120, 127 (1983); L. F. Abbott and P. Sikivie, Phys. Lett. B120, 133 (1983); M. Dine and W. Fischler, Phys. Lett. B120, 137 (1983).

15. J. E. Kim, Phys. Rev. D31, 1733 (1985); K. Choi and J. E. Kim, Phys. Rev. D32, 1828 (1985).

16. E. Witten, Phys. Lett. B149, 351 (1984); Phys. Lett. B153, 243 (1985).

17. J. E. Kim, JHEP 9905, 022 (1999).

18. P. Sikivie, Phys. Rev. Lett. 48, 1156 (1982); K. Choi and J. E. Kim, Phys. Rev. Lett. 55, 2637 (1985).

19. J. Ellis, J. E. Kim and D. V. Nanopoulos, Phys. Lett. B145, 181 (1984).

20. M. B. Green and J. H. Schwarz, Phys. Lett. 149, 117 (1984).

21. K. Choi and J. E. Kim, Phys. Lett. B154, 393 (1985).

22. E. Witten, Phys. Lett. 153, 243 (1985); K. Choi and J. E. Kim, Phys. Lett. B165, 71 (1985).

23. K. Choi, Phys. Rev. D56, 6658 (1997).

24. X. G. Wen and E. Witten, Phys. Lett. B166, 397 (1988).

25. Z. Lalak, S. Lavignac and H. P. Nilles, Nucl. Phys. B559, 48 (1999).

26. G. Lazarides, C. Panagiotakopoulos and Q. Shafi, Phys. Rev. Lett. 58, 1707 (1987).

27. H. Georgi, J. E. Kim and H. P. Nilles, Phys. Lett. B437, 325 (1998).

28. A. Patziris and K. Kang, Phys. Rev. D33, 3509 (1986).

29. K. Choi, K. Kang and J. E. Kim, Phys. Rev. Lett. 62, 849 (1989).

30. J. E. Kim, Phys. Rep. 150, 1 (1987).

31. P. Sikivie, Phys. Rev. Lett. 51, 1415 (1983); ibid 52, 695(E) (1984).

32. J. E. Kim, Phys. Rev. D58, 055006 (1998).

33. R. A. Battye and E. P. S. Shellard, Phys. Rev. Lett. 73, 2954 (1994).

34. S. Perlmutter et al, Astrophys. J. 483, 565 (1997).

35. J. E. Kim, hep-ph/9907528. 